\title{
Simulating Balance Recovery Responses to Trips Based on Biomechanical Principles
}

\author{
Takaaki Shiratori ${ }^{1,2}$ Brooke Coley ${ }^{3} \quad{\text { Rakié } \text { Cham }^{3} \quad \text { Jessica K. Hodgins }}^{1,2}$ \\ ${ }^{1}$ Carnegie Mellon University $\quad{ }^{2}$ Disney Research, Pittsburgh $\quad{ }^{3}$ University of Pittsburgh
}

\begin{abstract}
To realize the full potential of human simulations in interactive environments, we need controllers that have the ability to respond appropriately to unexpected events. In this paper, we create controllers for the trip recovery responses that occur during walking. Two strategies have been identified in human responses to tripping: impact from an obstacle during early swing leads to an elevating strategy, in which the swing leg is lifted over the obstacle and impact during late swing leads to a lowering strategy, in which a swing leg is positioned immediately in front of the obstacle and then the other leg is swung forward and positioned in front of the body to allow recovery from the fall. We design controllers for both strategies based on the available biomechanical literature and data captured from human subjects in the laboratory. We evaluate our controllers by comparing simulated results and actual responses obtained from a motion capture system.
\end{abstract}

Categories and Subject Descriptors (according to ACM CCS): I.3.7 [Computer Graphics]: Three-Dimensional Graphics and Realism—Animation; I.6.8 [Simulation and Modeling]: Types of Simulation—Reactive responses

\section{Introduction}

Because of its ability to respond to changing events, physical simulation has been put forward as a good source of motion for interactive systems such as video games. Simulation has been used very successfully to compute the motion of passive objects such as falling rocks or fracturing objects in video games, but simulating characters with a variety of behaviors has proved much more difficult. To leverage the potential of this technology, the character must be able to respond to unexpected external disturbances caused by events in the environment. Realistic responses to perturbations are credited with making a character appear to be alive rather than merely an automaton following prerecorded trajectories [FvdPT01, KKI02,ZH02, KLK04, ACSF07, MZS09].

In this paper, we create a human simulation with recovery responses to trips by modeling data captured from human subjects in the laboratory (Figure 1). Trips are induced when the swing leg strikes an obstacle that is inserted unexpectedly

E-mail: siratori@cs.cmu.edu, bcc4+@pitt.edu,chamr@upmc.edu, jkh@cs.cmu.edu
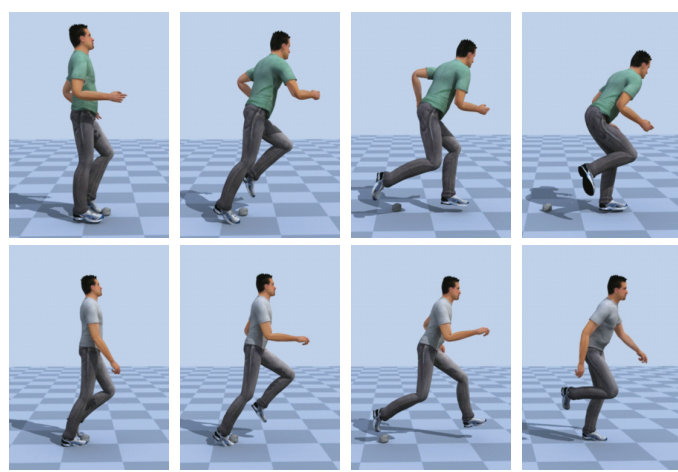

Figure 1: We create controllers to simulate balance recovery responses to trips (bottom) from biomechanical principles and motion capture data (top) we recorded in the laboratory.

into the walkway. The subject alters his or her leg trajectory significantly to avoid the obstacle and regain balance. In the biomechanical literature, such changes to the leg trajectories are categorized into two strategies: elevating and lowering 
strategies [EWP94] depending on whether the tripped leg is elevated to clear the obstacle or lowered and placed on the ground on the near side of the obstacle.

Our motivation for this research is two-fold. First, we would like to be able to create human characters with natural movement patterns that can respond appropriately to changes in their environment and recover (or not) from an unexpected trip. Second, we are interested in supplementing the data collected by biomechanical researchers and perhaps understanding better the responses to tripping and how those responses differ among various populations. Many biomechanics researchers have studied responses to trips because falls induced by tripping often cause severe injury, hospitalization, and disability for older adults [FCW98]. Appropriate and rapid postural response strategies are necessary to avoid these accidents. Experimental gait studies have been key in describing these responses, however, the causes of failed recovery attempts are difficult to determine from experiments alone because data is difficult to collect safely and subjects change their gait after having experienced a trip in the laboratory. We hope that computational modeling and simulation techniques such as those presented in this paper can complement experimental approaches to gain a greater understanding of trip-initiated postural responses and causes of falls.

Our models are based on principles for responses to trips described by biomechanical researchers [EWP94, SvWMD00, CKvdH04, PBvD04, PBvD05] and a dataset of trips that we recorded in the laboratory. According to the biomechanical literature, both the tripped and non-tripped legs operate based on states determined by foot and obstacle/ground contact. Therefore, we employ finite state machines (FSM) to model the responses. This FSM approach has also been used in computer graphics to construct controllers for running and other behaviors [RH91, HWBO95, YLvdP07]. The control laws for each state of the FSM are modeled from the motion capture data. We use a comparison between simulated results and motion capture data to show the effectiveness of our models.

The organization of this paper is as follows: Section 2 describes prior work done in the graphics community. Section 3 describes prior work on analysis of responses to trips in the biomechanics community, and also describes our laboratory setup for capturing responses to trips. Section 4 describes the dynamic simulation and controllers. Section 5 shows results of our models, and Section 6 concludes this paper by discussing the limitations of our method and possible future directions.

\section{Prior Work}

Research on physical simulation of human behaviors has been conducted by many researchers. Raibert and Hodgins first developed controllers that enabled a biped character to run [RH91]. Their idea was to design a finite state machine based on contact states between feet and ground and then design control actions for each joint in each state. This idea was extended to a running, vaulting, and diving human character by Hodgins and colleagues [HWBO95]. Yin and colleagues implemented FSM-based controllers for various behaviors such as a back flip and a skip [YLvdP07]. Our controllers also use FSMs to sequence the control actions for balance recovery responses to trips.

To enable physical controllers to simulate stylized motions such as a sneaky walk, researchers have focused on tracking motion capture data with a proportional-derivative (PD) controller. Because directly tracking motion capture data does not work for unstable behaviors due to differences between the person captured and the physical model, they modified the motion capture data with displacement mapping techniques to allow the simulated character to maintain its balance [SKL07]. They accomplished this goal by using linear time-varying approximations to estimate the balance strategy of the input stylized motion and by feedback error learning [YLvdP07, dSAP08, MLPP09]. To use these techniques to simulate responses to trips, we would need to collect a fair amount of data, because there is a wide variety in the responses to a trip based on timing of collision and its severity. Data is difficult to collect in this situation because each subject can only be tripped unexpectedly once.

The ability to simulate reactive responses to perturbations is very important because it is those natural responses to events that make the characters appear alive. One approach is to change the gains of PD controllers to deal with perturbations [ZH02, ACSF07]. For example, Zordan and Hodgins proposed a method to simulate a reactive motion from motion capture data by lowering the gains of the simulation during the perturbation, allowing the simulation to be pushed off the desired trajectory by the forces of the interaction, and then returning slowly to an appropriate motion capture trajectory [ZH02]. They also implemented a balancing controller that could maintain balance during the disturbances caused by the motion of the upper body. Another approach is to calculate appropriate torques based on a computation of the center of mass $(\mathrm{COM})$ and center of pressure (COP). Kudoh and colleagues considered a zero moment point (ZMP) [VBSS90] to optimize torques with which a character can react to a perturbation during stance [KKI02, KKI06]. The model of the stepping motion was based on insights in the biomechanical literature. Komura and colleagues modeled a single support phase during walking and running with an angular momentum-inducing inverted pendulum model based on ZMP and COM [KLK04]. Macchietto and colleagues proposed a method to adjust the COM and COP based on linear and angular momenta while tracking motion capture data [MZS09]. Though these techniques were able to synthesize realistic motions, we need a different approach for simulating a response to a trip; after the obstacle perturbs the swing leg, the character must change the leg trajectories 
significantly, because the obstacle remains in place and the foot must clear it to allow walking to continue.

Ye and Liu proposed a method to synthesize upper body motion reactive to a perturbation based on results in biomechanical literature [YL08]. They did not use physical simulation, but motion capture data to achieve their goal. Their method is applicable only to upper body motion and not to leg motions such as trips.

Boone and Hodgins designed controllers that allowed a running biped robot to react to trips and slips [BH04]. When the robot detected a slip, the slipping leg was immediately lifted allowing the robot to enter a flight phase and giving the controller time to prepare for safe landing on the slippery ground. When the robot detected a trip, the tripped or non-tripped leg immediately touched down on the obstacle or ground and allowed balance to be recovered. These strategies have some elements in common with those that have been found in human responses although Boone and Hodgins implemented them only on a two legged robot with telescoping legs rather than a full human model.

\section{Biomechanical Principles of Balance Recovery Responses to Trips}

According to Eng and colleagues [EWP94], there are two strategies for responses to trips. Impact from an obstacle during early swing leads to an elevating strategy, in which the leading (swing) leg is lifted over the obstacle (Figure 2 top). Impact during late swing leads to a lowering strategy, in which the swing leg is rapidly lowered to the ground in front of the obstacle and then the trailing leg is swung forward clearing the obstacle and positioned in front of the body to allow a recovery from the fall (Figure 2 bottom). In both strategies, the swing and support legs are used to arrest the forward angular momentum caused by the collision between the swing foot and an obstacle.

According to Schillings and colleagues [SvWMD00], the choice of strategies depends on the timing of the collision with an obstacle. When a collision is caused in early swing (5-25\% of stride duration in normal walking), the elevating strategy is chosen. And when the collision is caused in late swing (55-75\%), the lowering strategy is chosen. In mid swing (30-50\%), both strategies may occur.

\subsection{Common Reactions of Strategies}

Although the strategies appear to be quite different, two elements are common in the initial phase of the strategies.

Passive Reaction: In both strategies, a passive motion of the swing leg can be observed right after the collision with the obstacle. Schillings and colleagues observed this phenomenon in muscle responses obtained from electromyographic (EMG) activity [SvWMD00]. The swing ankle is plantar flexed first, and then the swing knee is flexed.

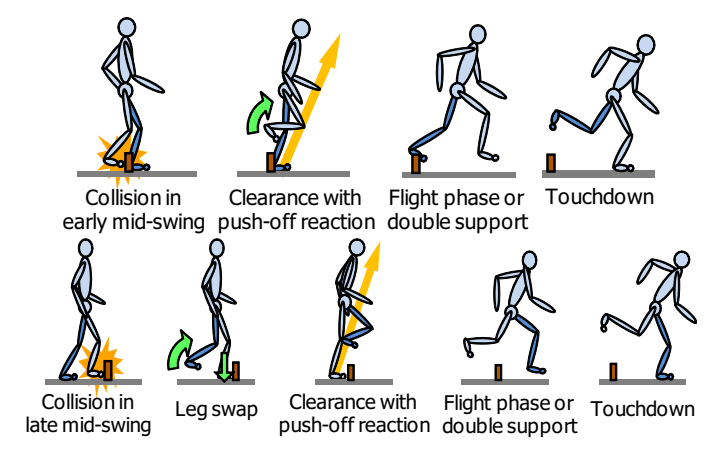

Figure 2: Responses to trips: elevating (top) and lowering (bottom) strategies.

Push-off Reaction of Support Leg: Pijnappels and colleagues found that the support leg is activated to strongly push against the ground right after the passive reaction [PBvD04], in an action called the push-off reaction. They later analyzed the reaction in detail to understand how an early push-off reaction assists in successful recovery [PBvD05]. In the push-off reaction, the support hip is extended by hamstring and gluteal bursts, and this hip extension moment can decelerate the forward angular velocity of the trunk. The timings of the bursts are around 50 msec and $80 \mathrm{msec}$ after the collision, respectively. This hamstring activity also results in flexion of the support knee, and then the quadriceps activity accelerates extension of the support knee around $120 \mathrm{msec}$ after the collision. Meanwhile, the rapid responses of the triceps surae works to apply plantar flexion torque to the ankle joint. This burst is observed around $75 \mathrm{msec}$ after the collision. The ground reaction forces derived from the push-off reaction are significantly larger in both horizontal and vertical directions than that in normal walking. Finally, the ground reaction forces decelerate the forward angular momentum of the body providing a longer stance duration to position the swing leg to clear the obstacle.

\subsection{Elevating Strategy}

The tripped swing leg acts as a recovery leg and is elevated to clear the tripping obstacle while the support leg creates the push-off reaction after the passive reaction. According to the analysis conducted by Schillings and colleagues [SvWMD00], first a large biceps femoris burst can be observed to flex the swing knee and to clear the obstacle, followed by a rectus femoris burst to extend the knee and to touch down beyond the obstacle. The timing of these bursts are around $64 \mathrm{msec}$ and $154 \mathrm{msec}$ after the collision, respectively. Regarding the swing ankle joint, a tibialis anterior burst can often be observed about $75 \mathrm{msec}$ after the collision and acts for ankle dorsiflexion. After the push-off reaction, a flight phase [PBvD04] or a double sup- 


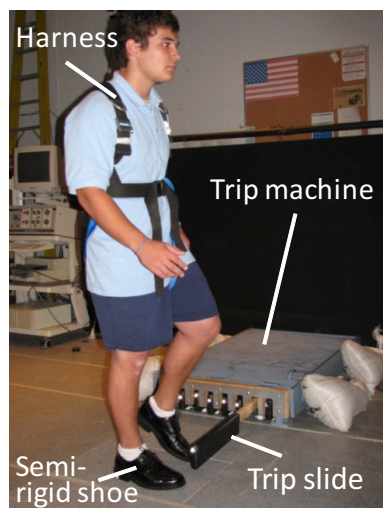

Figure 3: Capturing responses to trips in the laboratory. Trips were randomly inserted during several walking trials. Subjects had no knowledge of timing, placement or quantity of trips they would experience.

port phase [CKvdH04, CKvdH05] might occur followed by touchdown on the ground with the tripped leg.

\subsection{Lowering Strategy}

In the lowering strategy, a tripped swing leg touches down on the ground immediately after the passive reaction, and acts as a support leg for the push-off reaction due to a rectus femoris burst followed by a biceps femoris burst. These bursts occur with $62 \mathrm{msec}$ and $87 \mathrm{msec}$ latency, respectively [SvWMD00]. Simultaneously, tibilias anterior and soleus bursts can be observed with a latency of $40 \mathrm{msec}$ to achieve ankle dorsiflexion. Additionally, tibilias anterior bursts are observed again to achieve more ankle dorsiflexion around $60 \mathrm{msec}$ after the collision. After the tripped leg touches down, a soleus burst occurs again to achieve ankle plantar flexion for the push-off reaction. This phenomenon can be observed around $110 \mathrm{msec}$ after the collision. Also following the tripped leg touchdown, the non-tripped leg becomes the recovery leg, the leg clearing the obstacle. Then, similar to what is observed in the elevating strategy, a flight phase or double support phase might occur followed by touchdown on the ground with the non-tripped leg.

\subsection{Arm Motions during Responses to Trips}

Though arms do not play as critical a role as the legs during responses to trips, vigorous motions of arms can still be observed and do serve a purpose in trip recovery. From a biomechanical perspective, it is speculated that one function of arm motions is to protect body and head in the case of failure to recover balance and another is to increase the whole body moment of inertia and reduce the acceleration around the COM.

In both strategies, the arm contralateral to the swing leg
Table 1: Number and walking speeds of each strategy.

\begin{tabular}{|c|c|c|c|c|}
\hline & Elev. (DS) & Elev. (FL) & Lower. (DS) & Lower. (FL) \\
\hline Number & 4 & 3 & 4 & 3 \\
\hline Speed $[\mathrm{m} / \mathrm{s}]$ & 1.15 & 1.44 & 0.942 & 1.44 \\
$(\mathrm{SD})$ & $(0.146)$ & $(0.0751)$ & $(0.191)$ & $(0.232)$ \\
\hline
\end{tabular}

tends to move in the forward direction more than in normal walking, while the arm ipsilateral to the swing leg tends to move in a sideward direction rather than backward [RMKT08]. Specifically, the motion of the contralateral arm works to reduce the moment of inertia around the $\mathrm{COM}$ in the sagittal plane, and also elevates the COM position and increases the time that the swing leg can be positioned. The ipsilateral arm works to reduce the moment of inertia in the coronal plane, which provides stability in the coronal plane. Muscle activities inducing these arm motions can be observed within $100 \mathrm{msec}$ after trips [PWRvD08].

\subsection{Observing Trips}

We recruited nine young (21-35 year old) and seven older (65-75 years old) healthy adults, screened for neurological and musculoskeletal abnormalities, to measure their reactions to trips induced in the laboratory. We recorded the whole body motion of subjects at $120 \mathrm{~Hz}$ with a Vicon optical motion capture system. Figure 3 shows our experimental setup for recording trip responses. First, we asked subjects to walk on a known dry floor at a self-selected speed to retrieve baseline gait characteristics. Then, we informed subjects that in the next set of trials, at some point they would experience a trip. Subjects did not know the timing or place at which a trip would occur. Three trips were randomly inserted into five unperturbed trials. All subjects wore a harness to prevent injury in the event of an irrecoverable loss of balance. The trips were triggered at heel contact of the leading/right foot to catch the trailing/left foot in mid-swing. Only the first (unexpected) trip is used for analysis.

Table 1 summarizes our dataset of trip responses. Seven subjects performed an elevating strategy, seven performed a lowering strategy. One failed to recover balance, and we failed to obtain a trip motion for one subject, because the trip slide hit the foot from the side. In both strategies, responses with a double support phase or with a flight phase could be observed with the selection between those two responses being dependent upon walking speed.

We observed a wide variety of shoulder and elbow motions in all subjects. For example, one subject extended his elbows and abducted his shoulders, and another subject flexed his elbows to about $90^{\circ}$, and shoulder flexion angles were greater than in normal walking However, we observed the tendencies described by Roos and colleagues [RMKT08]. All the subjects moved the arm con- 

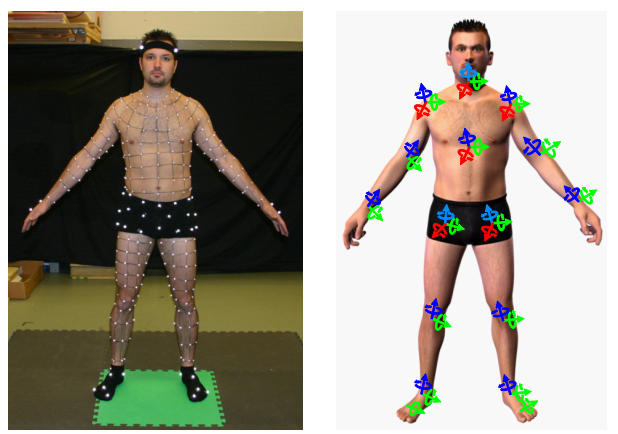

Figure 4: Our human model (right) is based on a skin model estimated from 350 optical markers (left). We first obtain the detailed human surface from 350 markers, and then calculate the volume of each body segment to estimate the mass and moment of inertia. This model has 16 joints with 36 internal DOFs (42 DOFs in total).

tralateral to the swing leg in the forward direction and moved the arm ipsilateral to the swing leg in the sideways direction.

\section{Simulation of Balance Recovery Responses}

Based on these biomechanical principles, we designed controllers to simulate responses to trips that occur during walking. We employed FSMs for each strategy similar to the traditional running and walking controllers used for other biped characters [RH91, YLvdP07, Hod91]. Torques are applied to each joint using a proportional-derivative (PD) controller:

$$
\tau=k_{p}\left(\theta_{d}-\theta\right)-k_{d} \dot{\theta},
$$

where $\theta_{d}$ is desired joint position, $\theta$ and $\dot{\theta}$ are the current joint angle and angular velocity, and $k_{p}$ and $k_{d}$ are spring and damper gains, respectively.

\subsection{Human Model}

We model a human character from detailed meshes obtained by Park and Hodgins [PH06]. Then, we calculate the volume of each body segment using Mirtich's method [Mir96], assuming that the density of the human body is equal to that of water. This model has 16 joints with 36 internal DOFs. All joints except for chest, neck, shoulders, hips and toes are modeled as a U-joint with two DOFs. The shoulder and hip joints are modeled as ball joints with three DOFs, and the toe joints are a pin joint with one DOF. The pelvis is uncontrolled and free to translate and rotate. The bottom of each foot has 96 contact points with the ground.

\subsection{Simulation Initialization}

The motion capture data of walking is kinematically played back until the time that the trip occurred (approximately the

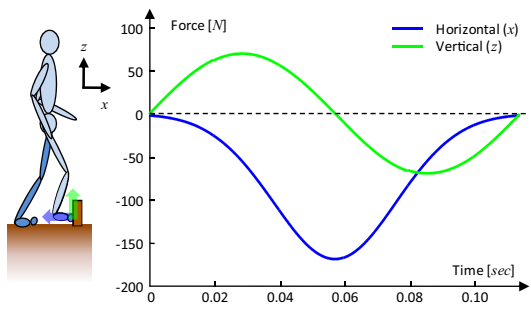

Figure 5: Our model of tripping forces.

middle of the swing phase). The simulation is initialized using the joint angles and velocities recorded in the motion capture data. If a support foot is above or under ground because of measurement errors in the motion capture or the skeletal estimation, we adjust the height of the pelvis so that the support leg is positioned on the ground. Ground reaction forces are calculated using the penalty method and applied to each contact point. The spring and damper coefficients are $3000 \mathrm{~N} / \mathrm{m}$ and $10 \mathrm{Ns} / \mathrm{m}$, respectively.

\subsection{Controller for Elevating Strategy}

The controller for the elevating strategy consists of four states: Passive Reaction, Clearance, Flight for control during a flight phase or Fall and Double Support for control during a double support phase, and Single Support After Trip. Figure 6 illustrates the state machine of the controllers.

In Passive Reaction, the character tries to maintain normal walking. The support leg maintains the torso attitude and keeps its stance posture [RH91, YLvdP07]. The swing leg moves forward to prepare for next step. Because the Passive Reaction occurs is in middle of the swing, no torque is applied to the swing knee [Per92]. Tripping forces are applied to the swing toe based on a model from the biomechanical literature. Based on tripping forces computed using inverse dynamics and force plates by Pijnappels and colleagues [PBvD04], we model the horizontal forces $F_{x}$ with a Gaussian function and the vertical forces $F_{z}$ with a sine curve:

$$
\begin{aligned}
& F_{x}(t)=-F_{x}^{\max } \exp \left(-\left(t-T_{c}\right)^{2} /\left(T_{\text {trip }} / 6.0\right)\right) \\
& F_{z}(t)=F_{z}^{\text {max }} \sin \left(2 \pi t / T_{\text {trip }}\right)
\end{aligned}
$$

where $F_{x}^{\max }$ is a maximum horizontal force of $170 \mathrm{~N}, F_{z}^{\max }$ is a maximum vertical force of $70 \mathrm{~N}, T_{\text {trip }}$ is a collision duration of $0.114 \mathrm{sec}$, and $T_{c}=0.5 * T_{\text {trip }}$, respectively. Figure 5 shows our model of tripping forces.

After a delay, the state is transitioned to Clearance. The delay differs for each joint, and the timings of the transitions are based on the muscle response timings described in Section 3.1. We add a constant offset of $50 \mathrm{msec}$, which is a reasonable approximation of the interval between muscle activation and torque generation [RTI76] to the muscle response timings. In Clearance, flexion torques are applied to 


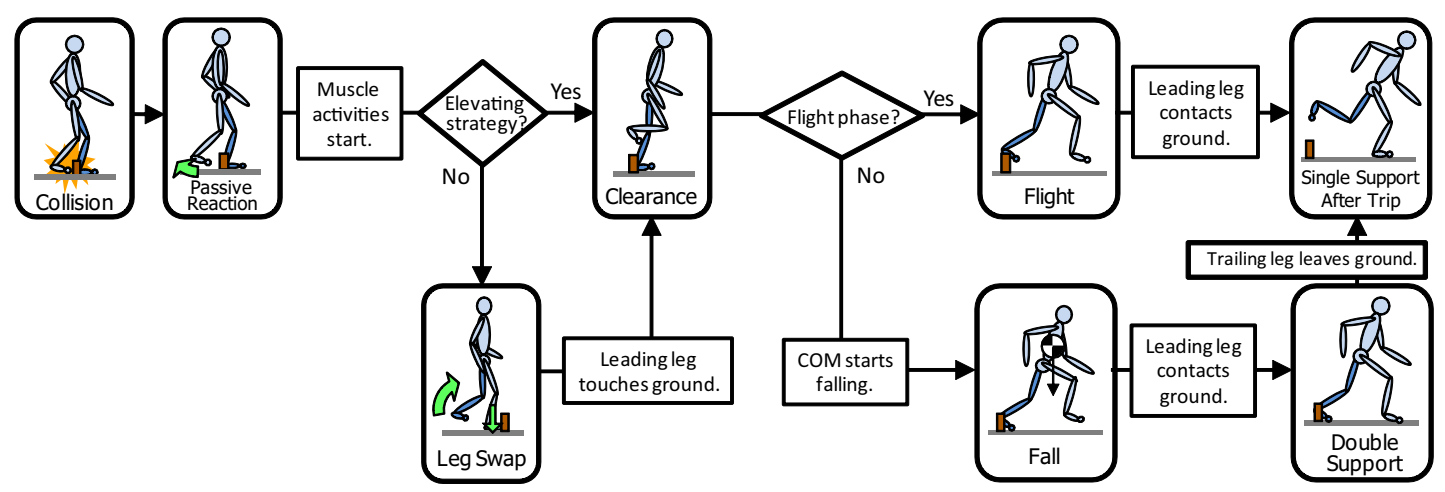

Figure 6: State machine of the controller for recovery from trips.

the swing leg to clear the obstacle and extension torques are applied to the support leg to perform a push-off reaction. A compensation torque, $\tau_{c}$, is also applied to the support ankle to prepare for the next Flight state. The compensation torque is calculated as

$$
\tau_{c}=k_{c}\left(v_{z}^{d}-v_{z}\right)
$$

where $k_{c}$ is a coefficient for the compensation and $v_{z}^{d}$ and $v_{z}$ are target and current vertical velocities of the COM, respectively. The coefficient for the compensation is set based on walking speed because that modeled our dataset well with higher coefficients for faster walking speeds.

If the stance leg leaves the ground during Clearance, the state is transitioned to Flight. In Flight, the character prepares for landing when the COM starts falling. The front leg is extended for ground contact, and the rear leg is flexed.

When the front leg contacts the ground, the state is transitioned to Single Support After Trip. The stance hip is then activated with torques that maintain torso attitude and the stance knee is extended, while the swing leg moves forward with a flexed posture.

If the stance leg does not leave the ground and the COM starts falling, the state becomes Fall, in which the front swing leg is extended for landing. When the front leg touches the ground, the state becomes Double Support. In Double Support, the front leg maintains the torso attitude and maintains its length. The rear leg is flexed and moves forward for the next step.

\subsection{Controller for Lowering Strategy}

The controller for a lowering strategy consists of five states: Passive Reaction, Leg Swap, Clearance, Flight for control during a flight phase or Fall and Double Support for control during a double support phase, and Single Support After Trip.

Passive Reaction in the lowering strategy is the same as that in the elevating strategy, and the same tripping forces are applied to the swing toe. After a short delay, the state changes to Leg Swap. In Leg Swap, extension torques are applied to the swing leg for ground contact, and the support leg maintains torso attitude. Once the swing leg touches the ground, the state becomes Clearance. The tripped leg becomes a support leg, and pushes against the ground to perform the push-off reaction. The non-tripped leg becomes a swing leg, and is flexed to clear the obstacle. The other states are the same as those in the controller of the elevating strategy.

\subsection{Parameters}

Except for the postures of a support leg during the push-off reaction, we set the target angles for each joint/state based on our motion dataset so that the angles achieved during the simulation are close to those observed in the dataset. For example, if the max angle of the leading knee for the Clearance is $120^{\circ}$ in motion capture data, we use $135^{\circ}$ for the target angle of the PD servo to prevent the need for very high gains for trajectory tracking. For support leg postures during the push-off reaction, we set target angles of the support hip, knee and ankle to $0^{\circ}, 5^{\circ}$ and $60^{\circ}$ so that the support leg is extended for the push-off reaction. For Fall, Double Support, Flight, and Single Support After Step states, target angles are empirically set so that balance is recovered with one step.

Gains of PD controllers for each joint are empirically tuned. Spring coefficients during Passive Reaction are $500 \mathrm{Nm} / \mathrm{rad}$ for the swing hip joint, $1000 \mathrm{Nm} / \mathrm{rad}$ for the support hip, $300 \mathrm{Nm} / \mathrm{rad}$ for the knee and ankle joints, and $100 \mathrm{Nm} / \mathrm{rad}$ for toe joints. After balance recovery starts, those parameters are increased by a factor of approximately 1.5. In Single Support After Trip, the spring coefficient for the support hip joint is around $1500 \mathrm{Nm} / \mathrm{rad}$, and those for other joints are mostly equal to those in Passive Reaction. We use $k_{d}=0.1 * k_{p}$ for damper gains. 

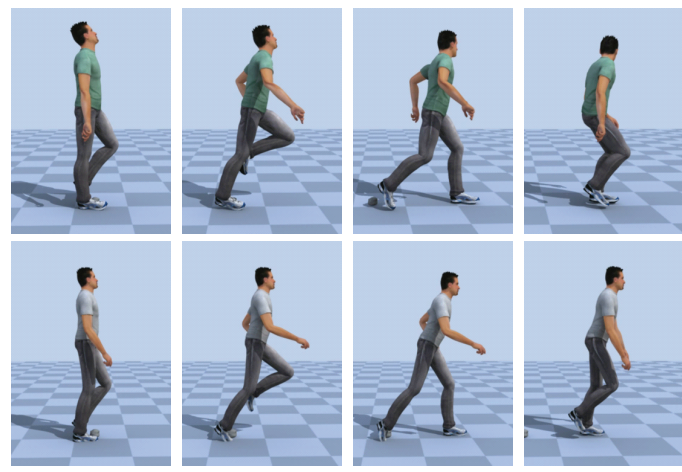

Figure 7: Results of simulating an elevating strategy with a double support phase. Top: motion capture data, and bottom: simulated results. From left to right: Passive Reaction, Clearance, Double Support, and Single Support After Step.

\subsection{Simulation of Upper Body Motion}

Because arm motions appear to differ significantly for each individual we recorded, it is difficult to model them with our current motion dataset. Therefore, we picked one motion trajectory in which the subject used the same strategy as in the current simulated motion, and used that trajectory as the target arm joint angles. We use the timing of shoulder responses reported by Pijnappels and colleagues [PWRvD08] and the offset between muscle activity and torque generation to activate the shoulders and elbows. After the front leg touches down and the state becomes Single Support After Trip, the arms are activated to perform swings similar to normal walking. We maintain the joint angles of the chest, neck and hands at the tripping instant with high gain PD controllers.

\section{Results}

We performed experiments with our controllers for tripping responses to determine how well they matched recorded data and how natural the resulting motion appeared. For each strategy, we used both slow and fast walking motion as inputs. These walking motions were captured from the subject whose detailed skin model was used to create the dynamic model. We set the coefficient and target vertical velocity of the COM of the compensation torque to 50 and $1.2 \mathrm{~m} / \mathrm{s}$ for the slow input walking, and 300 and $1.2 \mathrm{~m} / \mathrm{s}$ for fast input walking, respectively.

\subsection{Results of Simulating Elevating Strategy}

Figure 7 shows a comparison between the simulated result with double support and the motion capture data. The average walking speed of the input motion capture is $1.0 \mathrm{~m} / \mathrm{s}$. The simulated double support phase matches the captured data (third column).

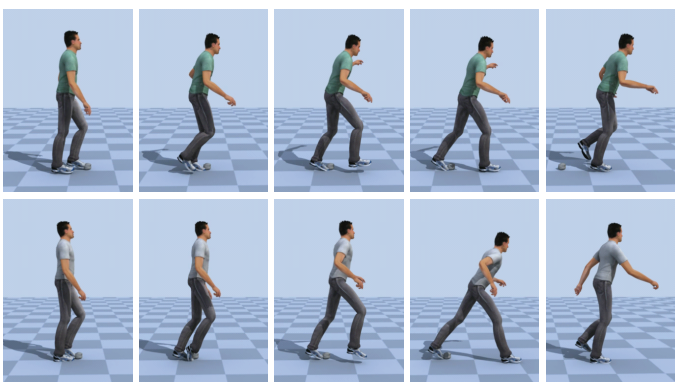

Figure 8: Results of simulating a lowering strategy with double support. Top: motion capture data, and bottom: simulated result. From left to right: Passive Reaction, Leg Swap, Clearance, Double Support, and Single Support After Step.

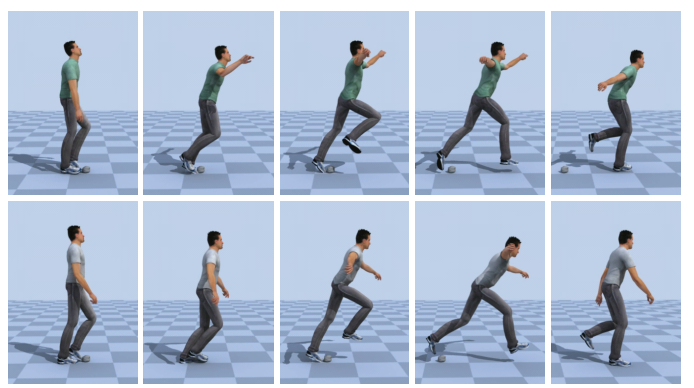

Figure 9: Results of simulating a lowering strategy with a flight phase. Top: motion capture data, and bottom: simulated result. From left to right: Passive Reaction, Leg Swap, Clearance, Flight, and Single Support After Step.

Figure 1 shows a comparison between the simulated result with a flight phase and motion capture data with a walking speed of $1.4 \mathrm{~m} / \mathrm{s}$.

For the elevating strategy with a double support phase, the duration from toe-off to heel-contact in normal walking was $0.45 \mathrm{sec}$, and in the recovery responses was $0.742 \mathrm{sec}(64 \%$ increase over normal walking). For the simulation, the corresponding durations were $0.40 \mathrm{sec}$ and $0.70 \mathrm{sec}(75 \%$ increase over normal walking). For the strategy with a flight phase, the duration of a stride in normal walking was $0.42 \mathrm{sec}$ vs. $0.59 \mathrm{sec}$ during a trip. For the simulation, normal walking was $0.3 \mathrm{sec}$ and tripping was $0.65 \mathrm{sec}$. These observations were similar to those made by Pijnappels and colleagues [PBvD05].

\subsection{Results of Simulating Lowering Strategy}

Figure 8 shows a comparison between the simulated result with double support and the motion capture data. The average walking speed of the input motion capture was $0.75 \mathrm{~m} / \mathrm{s}$. As with the elevating strategy, the double support phase was performed without falling down. 
Table 2: RMSEs between motion capture data and simulated results. The errors are calculated with pitch angles of each joint. The units are [deg/frame] and the frame rate is $120 \mathrm{~Hz}$.

\begin{tabular}{|c|c|c|c|c|}
\hline & Elev. (DS) & Elev. (FL) & Lower. (DS) & Lower. (FL) \\
\hline Pelvis & 5.80 & 12.9 & 15.7 & 27.6 \\
Lead. hip & 4.55 & 12.2 & 11.5 & 30.7 \\
Trail. hip & 6.99 & 16.1 & 10.0 & 18.2 \\
Lead. knee & 16.8 & 5.41 & 10.8 & 22.7 \\
Trail. knee & 14.4 & 18.0 & 10.9 & 39.9 \\
Lead. ankle & 15.7 & 10.0 & 19.5 & 18.9 \\
Trail. ankle & 24.0 & 10.0 & 21.7 & 16.9 \\
\hline
\end{tabular}

Figure 9 shows a comparison between the simulated result with a flight phase and the motion capture data. The input walking speed was $1.1 \mathrm{~m} / \mathrm{s}$.

For the lowering strategy with a double support phase, the duration from the trip to the ground contact of the recovery leg in the motion capture data was $0.54 \mathrm{sec}$, and that in the simulation was $0.70 \mathrm{sec}$. For the strategy with a flight phase, the corresponding duration was $0.57 \mathrm{sec}$ in the motion capture data and $0.72 \mathrm{sec}$ in the simulation. The simulation had a longer recovery motion than our dataset and those reported by Pavol and colleagues [POFG01]. However, the results appeared natural and looked similar to the motion capture data. as shown in the accompanying video.

\subsection{Quantitative Evaluation}

Figure 10 compares trajectories for the simulation and the motion capture of the leading foot's COM, and joint angle trajectories for the pelvis, leading hip, and knee. Table 2 shows root mean-square errors (RMSEs) of each joint between the simulated results and motion capture data used for setting the parameters of the simulations. For elevating strategies (top two rows), though the simulated foot was extended further and the pelvis pitch was less in the simulation than in the motion capture data, the resulting motion was qualitatively very similar to motion capture data. The pitch trajectories of the hip and knee of the simulation and the motion capture were similar. For lowering strategies (bottom two rows), the step length was longer and pelvis was pitched further in the simulations and, particularly, RMSEs of the lowering strategy with a flight phase are large, although overall the match seems reasonable given the variability in individual human responses to a trip.

\section{Discussion}

We designed our controllers to physically simulate balance recovery responses for trips. We first built a dynamic human model from detailed meshes obtained from a dense marker set. We then constructed finite state machines and corresponding control laws for each strategy based on observations in the biomechanical literature and data captured in our laboratory. We performed experiments to validate our controllers by changing walking speed. The quantitative evaluations showed that our models were reliable, generating both elevating and lowering strategies at a variety of different speeds and initial conditions. Although the current controllers can simulate only responses to trips, we believe that it should be possible to integrate these controllers with controllers for walking or to blend with motion capture data before and after the trip to create a character that can move freely around the environment, responding to unexpected trips when they occur.

Through these experiments, we believe that we have demonstrated that the FSMs for our controllers are quite general. In particular, the criteria for changing states, which is derived from contact states and muscle responses, and the functions of the support leg during the push-off reaction appear to generalize. However, other parameters, particularly the proportional gains of each joint/state and the trajectories for the limbs, were tuned manually. With a larger database of tripping trials, we might be able to more automatically determine the target angles for each state/joint. EMG data was recorded with our experiments and analysis of that data might help provide us with effective ways to design the proportional gains.

Another limitation of this work is the ground contact model. Complicated interactions between the feet and ground are observed. To capture the interactions, we need a larger marker set which can measure deformations of the semi-rigid shoes or feet very precisely, and more precise ground reaction forces by using larger or more force plates. In the work reported here, we use a pre-determined model of tripping forces reported in the biomechanical literature. If we were to use dynamic simulation for the forces with a precise ground contact model, we might be able to achieve more realistic simulations.

In order to generalize our controllers further, we need to collect more data from subjects in the laboratory. Though the controllers worked well in most cases, we could not achieve balance recoveries for severe trips. In our collected data, some of the severe trips were followed by a number of steps that included flight phases (mimicking slow running). Controllers that permit multiple steps could make the resulting trajectories and RMSEs closer to motion capture data. Additionally, timing of the collision is important for each strategy. Though automatic selection of the strategies can be easily achieved [SvWMD00], our controllers might not be able to simulate balance reactions to trips occurring in the early/late swing, because the controllers are based on motion capture data of reactions to trips occurring around the middle of the foot swing.

A future potential application of this work is to understand what factors are important for successful recovery responses to trips. One out of three adults who are over 65 years of age falls once a year, and in most cases this is caused by a 

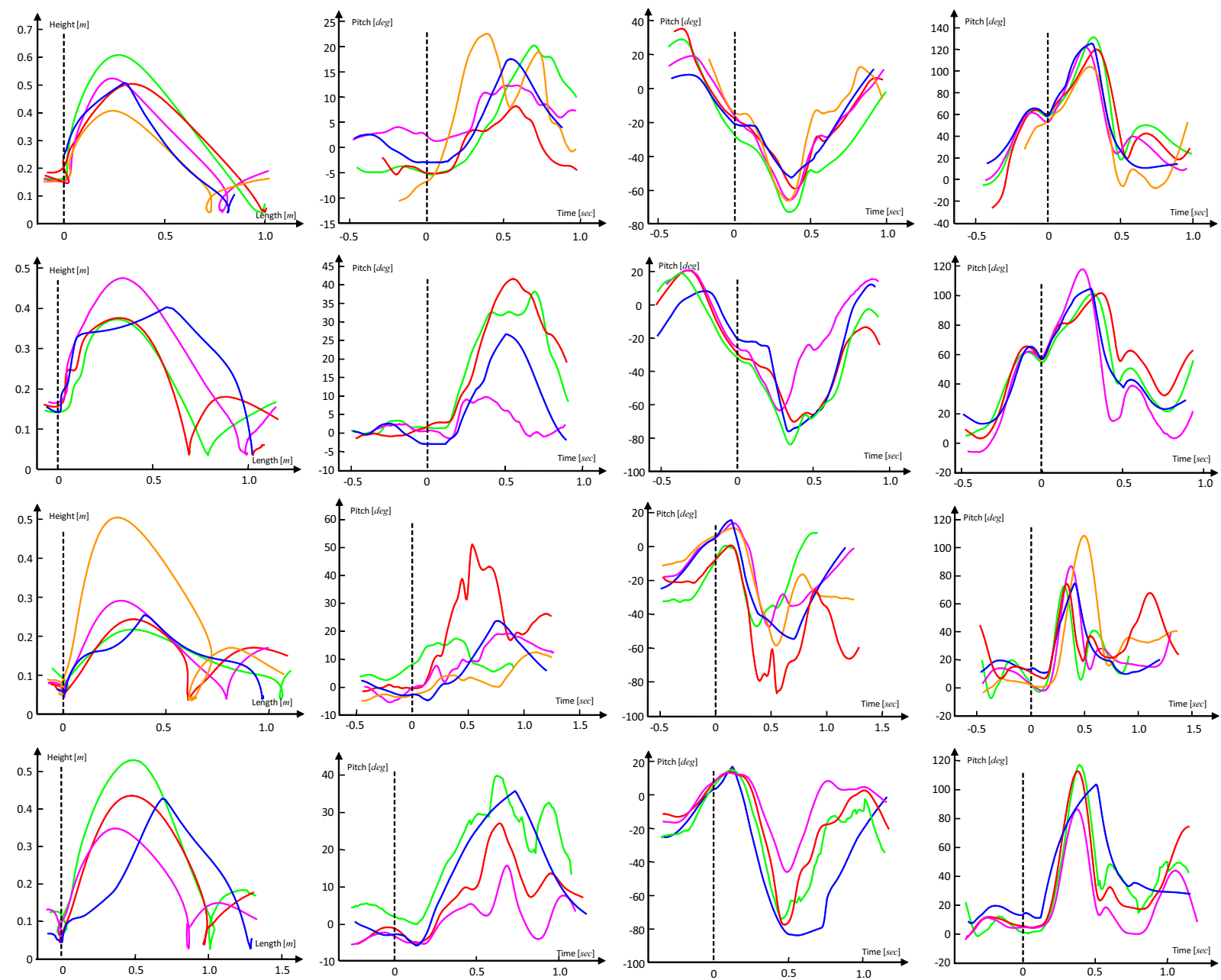

Figure 10: Comparison of trajectories of the leading foot's COM, and pitch trajectories of pelvis, hip, and knee (from left to right) in the simulated elevating strategy with double support and flight phases and the simulated lowering strategy with double support and flight phases (from top to bottom). Blue lines are the simulated results and others are the motion capture data. Vertical dashed lines indicate the position/timings of the trip.

trip or slip [NCH91, BAMT97]. Therefore, revealing what factors are important for balance recovery after trips might allow a reduction in the risk of falls for older people. However, experimental data is limited because of the difficulty of collection and the artificial nature of gait performed in the laboratory. If we could develop truly general control models for recovery after trips, we would be able to simulate responses under various conditions and test hypotheses such as slow timing of muscle responses or lower muscle tone. In this way, generalized controllers might be able to provide the scientific underpinning for techniques for training and rehabilitation for older adults.

\section{Acknowledgements}

We would like to thank Adam Bargteil for providing us with his codes for calculating mass and moment of inertia from polygonal data, Moshe Mahler for creating the character and video editing, and Justin Macey for his help modeling the character. We also thank Autodesk for their donation of the 3D animation and rendering package Maya. This study is funded in part by F31 AG025684-03 NIH Ruth L. Kirschstein Award and by the NSF-EEC-0540865 Quality of Life Technology Engineering Research Center.

\section{References}

[ACSF07] Allen B., Chu D., Shapiro A., Faloutsos P.: On the beat! timing and tension for dynamic characters. In Proc. 
ACM SIGGRAPH/Eurographics Symposium on Computer Animation (2007), pp. 239-247.

[BAmT97] Berg W. P., Alessio H. M., Mills E. M., Tong C.: Circumstances and consequences of falls in independent community-dwelling older adults. Age \& Ageing 26 (1997), 261268.

[BH04] Boone G. N., Hodgins J. K.: Slipping and tripping reflexes for bipedal robots. Autonomous Robots 4, 3 (2004), 259271.

[CKvdH04] Cordero A. F., Koopman H. J. F. M., VAn DER HeLM F. C. T.: Mechanical model of the recovery from stumbling. Biological Cybernetics 91 (2004), 212-220.

[CKvdH05] Cordero A. F., Koopman H. J. F. M., van DeR Helm F. C. T.: Energy analysis of human stumbling: the limitations of recovery. Gait \& Posture 21, 3 (2005), 243-254.

[dSAP08] DA Silva M., ABE Y., Popović J.: Simulation of human motion data using short-horizon model-predictive control. Computer Graphics Forum 27, 2 (2008), 371-380.

[EWP94] Eng J. J., Winter D. A., Patla A. E.: Strategies for recovery from a trip in early and late swing during human walking. Experimental Brain Research 102 (1994), 339-349.

[FCW98] Fingerhut L. A., COX C. S., WARnER M.: International comparative analysis of injury mortality: Findings from the ice on injury statistics, 1998. National Center for Health Statistics, Hyatsville, MD.

[FvdPT01] Faloutsos P., van De Panne M., Terzopoulos D.: Composable controllers for physics-based character animation. In Proc. ACM SIGGRAPH 2001 (2001), pp. 251-260.

[Hod91] Hodgins J. K.: Biped gait transitions. In Proc. IEEE Int'l Conf. on Robotics and Automation (1991), pp. 2092-2097.

[HWBO95] Hodgins J. K., Wooten W. L., Brogan D. C., O'BRIEN J. F.: Animating human athletics. In Proc. SIGGRAPH 1995 (1995), pp. 71-78.

[KKI02] Kudoh S., Komura T., IKeUCHI K.: The dynamic postural adjustment with the quadratic programming method. In Proc. IEEE/RSJ International Conference on Intelligent Robots and Systems (2002), pp. 2563-2568.

[KKI06] KUDOH S., KomURA T., IKEUCHI K.: Stepping motion for a human-like character to maintain balance against large perturbations. In Proc. IEEE International Conference on Robotics and Automation (2006), pp. 2661-2666.

[KLK04] Komura T., LeUng H., KuffNer J.: Animating reactive motions for biped locomotion. In Proc. ACM Symposium on Virtual Reality Software and Technology (2004), pp. 32-40.

[Mir96] MiRTich B.: Fast and accurate computation of polyhedral mass properties. Journal of Graphics Tools 1, 2 (1996), 31-50.

[MLPP09] Muico U., LeE Y., Popović J., Popović Z.: Contact-aware nonlinear control of dynamic characters. ACM Trans. on Graphics 28, 3 (2009).

[MZS09] Macchietto A., Zordan V., Shelton C. R.: Momentum control for balance. ACM Trans. on Graphics 28, 3 (2009).

[NCH91] NevitT M. C., Cummings S. R., Hudes E. S.: Risk factors for injurious falls: a prospective study. Journal of Gerontology A Biological Science \& Medical Science 46 (1991), M164-170.

[PBvD04] PiJnappels M., BobBert M. F., VAn DieËN J. H.: Contribution of the support limb in control of angular momentum after tripping. Journal of Biomechanics 37 (2004), 1811-1818.
[PBvD05] PiJnAPPEls M., BobBert M. F., VAN DiEËN J. H.: How early reactions in the support limb contribute to balance recovery after tripping. Journal of Biomechanics 38 (2005), 627634.

[Per92] PERry J.: Gait Analysis: Normal and Pathological Function. Slack Inc, 1992.

[PH06] PARK S. I., Hodgins J. K.: Capturing and animating skin deformation in human motion. ACM Trans. on Graphics 25 , 3 (2006), 881-889.

[POFG01] Pavol M. J., Owings T. M., Foley K. T., GRABINER M. D.: Mechanisms leading to a fall from an induced trip in healthy older adults. Journal of Gerontology: Medical Sciences 56A, 7 (2001), 428-437.

[PWRvD08] Pijnappels M., Wezenberg D., Reurink G., VAN DIEËN J. H.: The contribution of arm movements to balance recovery after a trip. In Proc. International Symposium on slips, trips and falls (2008).

[RH91] RAIBERT M. H., Hodgins J. K.: Animation of dynamic legged locomotion. In Proc. ACM SIGGRAPH 1991 (1991), pp. 349-358.

[RMKT08] Roos P. E., McGuigan M. P., Kerwin D. G., TREWARTHA G.: The role of arm movement in early trip recovery in younger and older adults. Gait \& Posture 27 (2008), 352-356.

[RTI76] Ralston H. J., Todd F. N., InMan V. T.: Comparison of electrical activity and duration of tension in the human rectus femoris muscle. Electromyography and Clinical Neurophysiology 16 (1976), 277-286.

[SKL07] SoK K. W., KIM M., LEE J.: Simulating biped behaviors from human motion data. ACM Trans. on Graphics 26, 3 (2007).

[SvWMD00] Schillings A. M., van Wezel B. M. H., MulDER T. H., DUYSENS J.: Muscular responses and movement strategies during stumbling over obstacles. Journal of Neurophysiology 83 (2000), 2093-2102.

[VBSS90] Vukobratović M., Borovac B., Suria D., StoKIC D.: Biped Locomotion: Dynamics, Stability, Control and Application. 7 of Scientific Fundamentals of Robotics, SpringerVerlag, 1990.

[YL08] YE Y., LIU C. K.: Animating responsive characters with dynamic constraints in near-unactuated coordinates. ACM Trans. on Graphics 27, 5 (2008), 112-116.

[YLvdP07] Yin K., LoKen K., VAN DE PANNE M.: SIMBICON: Simple biped locomotion control. ACM Trans. on Graphics 26, 3 (2007).

[ZH02] Zordan V. B., Hodgins J. K.: Motion capturedriven simulations that hit and react. In Proc. ACM SIGGRAPH/Eurographics Symposium on Computer Animation (2002), pp. 89-96. 